\title{
The influence of surface roughness in X-ray resonant magnetic reflectivity experiments
}

\author{
A. Verna ${ }^{1, a}$, B.A. Davidson ${ }^{1}$, A. Mirone $^{2}$, and S. Nannarone ${ }^{1,3}$ \\ 1 CNR-INFM TASC National Laboratory, Area Science Park-Basovizza, 34149 Trieste, Italy \\ 2 European Synchrotron Radiation Facility, 6 rue Jules Horowitz, Bote Postale 220, \\ 38043 Grenoble, France \\ 3 Dipartimento di Ingegneria dei Materiali e dell'Ambiente, Università di Modena e Reggio \\ Emilia, via Vignolese 905, 41100 Modena, Italy
}

Received 26 November 2011 / Received in final form 23 March 2012

Published online 15 June 2012

\begin{abstract}
We present simulations of X-ray resonant magnetic reflectivity (XRMR) spectra of the surface magnetic dead layer in $\mathrm{La}_{1-x} \mathrm{Sr}_{x} \mathrm{MnO}_{3}$ (LSMO) films that take in account the effect of different forms of roughness that can be encountered experimentally. The results demonstrate a method to distinguish between surface (morphological) roughness, and two generic kinds of magnetic roughness at the buried interface between the surface dead layer and the fully magnetic bulk part of the film. We show that the XRMR technique can distinguish between different types of magnetic roughness at the dead layer/bulk interface only if the sample surface is nearly atomically flat (the morphological roughness is one unit cell or less). Furthermore, to distinguish between the two types of magnetic roughness, the simulations show that fitting of XRMR spectra out to very high incidence angles must be performed. In the specific case of LSMO films with a dead layer with average thickness of 4 unit cells, this corresponds to an incidence angle $>50^{\circ}$.
\end{abstract}

\section{Introduction}

It is well known that the ferromagnetic properties of a material weaken in the presence of reduced dimensions due to the lack of exchange interactions for the atoms that are present near the boundary of the ferromagnetic system $[1,2]$. This has important consequences on the performance of spintronic devices, especially as they are miniaturized and the surface to volume ratio increases. Because the variation of the magnetic moment due to the breaking of crystalline symmetry at surface or interface extends for a depth of few unit cells (a unit cell in perovskite oxides typically has a lattice parameter $\sim 4 \AA$ ), for a spectroscopic investigation of the magnetism, a probe with a wavelength comparable to this dimension and sensitive to atomic magnetic moments is needed. The two most common techniques for measuring magnetic

a e-mail: verna@tasc.infm.it 
depth profiles on the unit-cell scale are reflectivity measurements by polarized neutrons $[3,4]$ and $\mathrm{X}$-rays in resonance condition with the magnetic species [5-10]. The advantage of X-rays produced e.g. by a synchrotron source in the soft X-ray regime is tunability over a range of $\mathrm{L}_{2,3}$ absorption edge energies of the transition metal ions (400-1000 eV), thus providing elemental sensitivity.

Reflectivity measurements as a function of the grazing angle (and thus as a function of the exchanged momentum perpendicular to sample surface) gives information about the distribution of a given quantity as a function of depth: density of electron charge in conventional out-of resonance X-ray reflectivity, distribution of atomic species, magnetic moment and other element-specific properties in resonant conditions. In the case of X-ray resonant magnetic reflectivity (XRMR), specular scattering measurements as a function of photon energy across the relevant absorption edge(s) of the magnetic element(s) are performed, using both right- and left-circularly polarized ( $R^{+}$and $R^{-}$, respectively) photons. Any difference between the values of reflectivity obtained by $R^{+}$and $R^{-}$light is called XRMR signal, and is due to the presence of a magnetic moment parallel to the scattering plane. XRMR signal as a function of photon energy is then collected at different grazing angles. As has been shown previously, oscillation in the sign of the XRMR spectra for different angle indicates inhomogeneity in the distribution of magnetic moments as a function of depth $[5,6,10]$ that, for these materials, can be ascribed to the presence of dead layers at the interfaces. The period of these oscillations gives a measure of the dead layer thickness $[5,6,11]$, and are usually discussed assuming an atomically flat surface and step-function like dead layer profile [5].

Real samples can exhibit roughness that can be either chemical, morphological or magnetic, and as we show here any of these can substantially modify the XRMR signal [6,9,12-14]. Experimentally, morphological roughness can be independently determined e.g. by scanning probe techniques such as atomic force microscopy. Independent determination of the surface roughness obviates its use as a fitting parameter during the analysis of the angular dependence of XRMR spectra. Chemical uniformity of the sample surface region can also be independently estimated by different surface-sensitive spectroscopic techniques and this is equally as important in simplifying the XRMR analysis.

If these two types of roughness are independently determined, then XRMR analysis can uniquely identify the behavior of the magnetic interface that defines the dead layer, i.e. the depth and sharpness of the magnetic/nonmagnetic boundary including magnetic roughness. The magnetization can either drop sharply moving from the bulk at a given distance from the interface (a sharp dead layer of definite thickness) or it can have a graded profile, in which the magnetization is gradually reduced from its bulk value far from the interface to zero or to a reduced finite value, over a distance of several unit cells. The later case is of particular interest in devices such as magnetic tunnel junctions, since a sufficiently gradual decrease towards the surface may permit a residual magnetization in the last monolayer at the interface, allowing for the production of a measurable spin-polarized current across the barrier in spite of dead-layer effects. It is also possible that roughness in the dead layer boundary ("magnetic roughness") can exist in spite of a flat sample surface morphology.

Here we analyze the XRMR signal from a thick sample that is chemically uniform but can have a finite surface roughness. Defining $\mathrm{z}$ as the distance from the vacuum/sample interface, the goal is to calculate the magnetic moment per atom $M(z)$ as a function of depth with a resolution of one unit cell from the experimentally measured XRMR spectra. A gradual variation in $M(z)$ can be ascribed not only to the presence of a definite magnetic profile but also to structural disorder, for example the presence of roughness at the interface. The different behaviors can be separated into three different cases, as shown in Fig. 1. In the first case, Fig. 1(a), the sample surface 


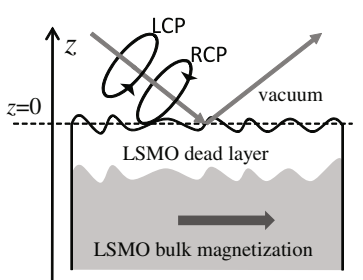

(a)

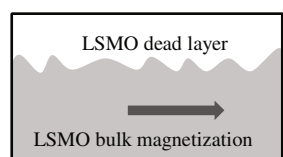

(b)

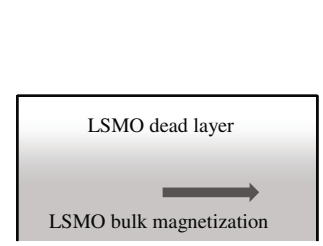

(c)

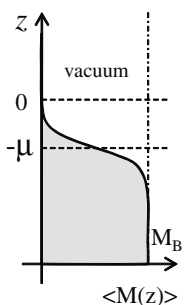

Fig. 1. A scheme for the three different systems under analysis: (a) An LSMO sample with a surface roughness that is perfectly reproduced at a the magnetic interface at a distance $z$ from the surface. The magnetic interface separates a nonmagnetized "dead layer" from the fully magnetized bulk. (b) A flat LSMO sample with a rough magnetic interface at an average distance $t$ from the surface. (c) A flat LSMO sample with graded magnetic profile that is function of the distance $-z$ from the surface. The averaged magnetic moment as a function of $z\langle M(z)\rangle$ is also shown.

has a finite roughness, and the magnetization is characterized by the same root mean square (rms) roughness as the surface morphology that is centered at a fixed depth from the surface plane $(z=0)$. In the second case, Fig. 1(b), the sample surface is atomically flat, but the depth of the magnetic/nonmagnetic boundary is different at different points $(x, y)$ of sample surface (magnetic roughness is present, in spite of a chemically and morphologically smooth surface). In the third case, Fig. 1(c), the sample is morphologically smooth but the magnetization profile is gradual along $z$ (and uniform along $x$ and $y$ for fixed $z$ ). If we consider the average value $\langle M(z)\rangle$ calculated on the different points $(x, y)$ at a fixed depth $z$, in these three cases we have a graded magnetic profile due to different origins.

In this paper we present simulation of XRMR measurements on a infinitely thick $\mathrm{La}_{1-x} \mathrm{Sr}_{x} \mathrm{MnO}_{3}$ film with a bare surface in order to verify how dichroic reflectivity measurements can distinguish between these three different cases of magnetic and structural "roughness". Defining $R^{+}$and $R^{-}$as the reflectivities from right- and leftcircularly polarized radiation, respectively, we will show that the average reflectivity $\bar{R}=\left(R^{+}+R^{-}\right) / 2$ and the difference (or dichroic) reflectivity $\Delta R=\left(R^{+}-R^{-}\right)$ are influenced differently by the different forms of roughness identified in Fig. 1, and that these are readily seen in the experimentally measured spectra, allowing the three cases to be identified.

\section{Simulations}

The sample is an (001) $\mathrm{La}_{0.7} \mathrm{Sr}_{0.3} \mathrm{MnO}_{3}$ (LSMO) film whose thickness is very large with respect to the absorption length of photons with energy near the $\mathrm{Mn} \mathrm{L}_{2,3}$ resonance $(640 \mathrm{eV})$, magnetized in the scattering plane with a total magnetic moment of 3.7 Bohr magnetons per Mn atom. The system presents two interfaces, the first between the LSMO film and a vacuum, and a magnetic interface due to the variation of the magnetization state of the film as a function of depth $z$. We assume that the dead layer has a thickness of 4 unit cells (in Fig. 1(c), this corresponds to the midpoint of the graded profile). The nonuniform boundaries are described by the rms roughness $\sigma$, and the point $z=0$ corresponds to the average height of the sample surface. We set the out-of-plane lattice parameter of LSMO to $3.83 \AA$, that is the $\langle 001\rangle$ LSMO lattice parameter when epitaxially grown on (001) $\mathrm{SrTiO}_{3}$ substrate by molecular beam epitaxy [15]. 
All the simulations have been performed using the Pythonic Programming for Multilayer (PPM) code by Alessandro Mirone [16]. The code is based on the $4 \times 4$ classical matrix formalism $[17,18]$ used to describe the propagation of the electromagnetic field into a multilayer formed by anisotropic components. Layers with identical chemical and structural composition but with different magnetic moment are described by different optical properties. In every layer four different eigenmodes of propagation are present, and their contribution to the total electromagnetic fields is determined by the condition of continuity for the electric and magnetic field at the interfaces. Roughness at the different interfaces is taken into account through an extension of the Croce-Nevot approach $[19,20]$ to the case of anisotropic media.

In the first two cases for roughness shown in Fig. 1(a) and (b), the sharp interface between the fully magnetized and not magnetized layer is present at different depth values $z$ for different $(x, y)$ points. The distribution of the $z$ values at which the interface is present is given by the gaussian function

$$
G(z ; \mu, \sigma)=\frac{1}{\sqrt{2 \pi \sigma^{2}}} e^{-\frac{(z-\mu)^{2}}{2 \sigma^{2}}}
$$

where $\sigma$ is the rms roughness and $\mu$ is the average value of the dead layer thickness, assumed 4 unit cells (uc) here. Because the interface is sharp, the average magnetic moment as a function of $z,\langle M(z)\rangle$ is given by the bulk magnetization $M_{B}$ multiplied by 1 minus the cumulative of the gaussian function

$$
\langle M(z)\rangle=M_{B}\left(1-\int_{-\infty}^{z} G\left(z^{\prime} ; \mu, \sigma\right) d z^{\prime}\right)=\frac{1}{2} M_{B}\left(\operatorname{erf}\left(\frac{\mu-z}{\sqrt{2} \sigma}\right)+1\right),
$$

where the error function is defined

$$
\operatorname{erf}(x)=\int_{0}^{x} e^{-y^{2}} d y
$$

In order to compare quantitatively the case of the graded magnetic profile 1 (c) with the cases $1(\mathrm{a})$ and (b), the magnetic profile is of the type indicated by equation (2) but with the difference that in this case $M(z)$ is not a statistical average but instead a constant magnetization for each depth $z$. Figure 2 illustrates the magnetization profile for case $1(\mathrm{c})$ given by equation (2) with $\langle M(z)\rangle=M(z)$ for various values of the smoothing parameter $\sigma$.

Because the (001) manganite perovskite consists of individual planes of $\mathrm{MnO}_{2}$ separated by $3.83 \AA$, the simulation divides the LSMO sample in 16 layers each $1 \mathrm{uc}$ thick, and the profile has discrete variations of the magnetic moments corresponding to each successive unit cell. The magnetic moment is homogeneous inside a layer. Below the 16th monolayer, a semi-infinite LSMO film with bulk magnetization $M_{B}$ is present.

We perform simulations of the reflectivity of right and left circularly polarized radiation as a function of the photon energy $E$ (across the $\mathrm{Mn}_{2,3}$ absorption edge) and of the grazing angle $\theta$ (between $1^{\circ}$ and $90^{\circ}$ ). In this way we construct an energyexchanged momentum map of the reflectivity from the sample.

In order to present the results in a compact and intuitive way, the integrals in energy of the average $(\bar{R})$ and dichroic $(\Delta R)$ reflectivities over the $\mathrm{L}_{3}$ resonance peak at fixed grazing angle are calculated:

$$
I_{L_{3}}(\bar{R})=\int_{L_{3}} \bar{R}(E, \theta) d E, \quad I_{L_{3}}(\Delta R)=\int_{L_{3}} \Delta R(E, \theta) d E,
$$

as well as their ratio $I_{L_{3}}(\Delta R) / I_{L_{3}}(\bar{R})$. 


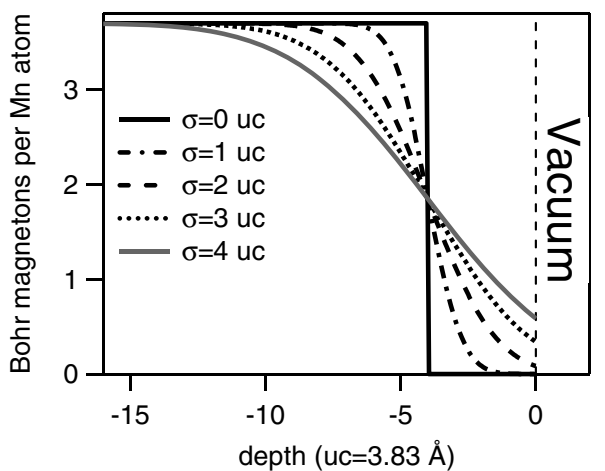

Fig. 2. Magnetic profiles for the graded profile illustrated in Fig. 1(c) for $\mu=4 \mathrm{uc}, M_{B}=$ $3.7 \mu_{B}$ and various values of the smoothing parameter $\sigma$. The vacuum/sample interface is at $z=0$.

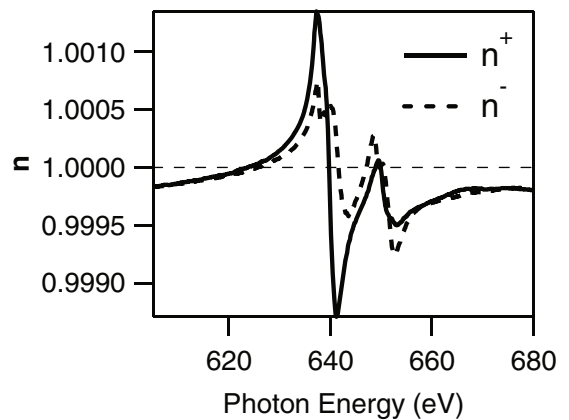

(a)

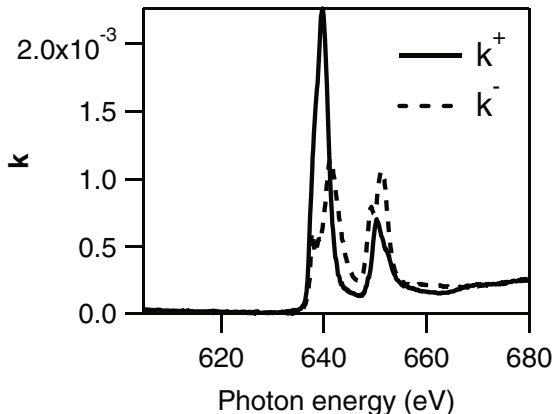

(b)

Fig. 3. (a) Real $n$ and (b) imaginary part $\kappa$ of the refractive index $\tilde{n}=n+i \kappa$ for photon helicity respectively parallel and antiparallel to the sample magnetization.

The simulation are performed using, as input values for the imaginary part $\kappa(E)$ of the complex refractive index $\tilde{n}(E)=n(E)+i \kappa(E)$, the total electron yield absorption spectra obtained on a $\mathrm{La}_{0.7} \mathrm{Sr}_{0.3} \mathrm{MnO}_{3}$ thin film epitaxially grown on a $\mathrm{SrTiO}_{3}$ substrate. Absorption measurements have been performed for the two opposite circular polarizations and are shown in Fig. 3(b). Magnetic circular dichroism sum rules [21] are used in order to normalize the difference between absorption spectra so that it corresponds to a total magnetic moment per Mn atoms of 3.7 Bohr magnetons per $\mathrm{Mn}$ atom. The real part $n$ of the refractive index used for the simulation is reported in Fig. 3(a) and is obtained from the imaginary part through Kramers-Krönig transforms [22]. We ignore in this simulation quadratic and higher-order magneto-optical effects.

The limits of the $\mathrm{L}_{3}$ peak are chosen as $621.0 \mathrm{eV}$ and $646.8 \mathrm{eV}$. The lower limit at $621 \mathrm{eV}$ has been chosen because for this value the $\Delta R$ quantity is always zero.

The upper value at $646.8 \mathrm{eV}$ is chosen because it corresponds to the minimum in absorption between the $\mathrm{L}_{3}$ and $\mathrm{L}_{2}$ peaks.

\section{Results}

The case shown in Fig. 1(a) assumes the presence of correlated surface and magnetic roughness. Simulations of the average and normalized dichroic reflectivities were performed for a range of rms roughness $\sigma$ from 0 to $4 \mathrm{uc}$. The integrals $I_{L_{3}}(\bar{R})$ and 
a)

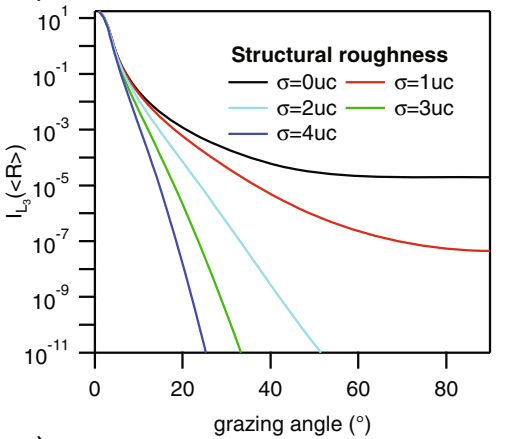

c)

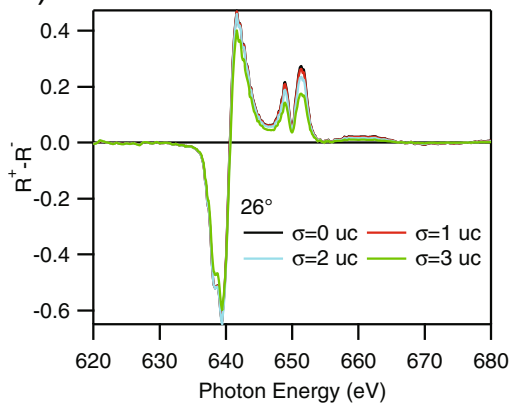

b)

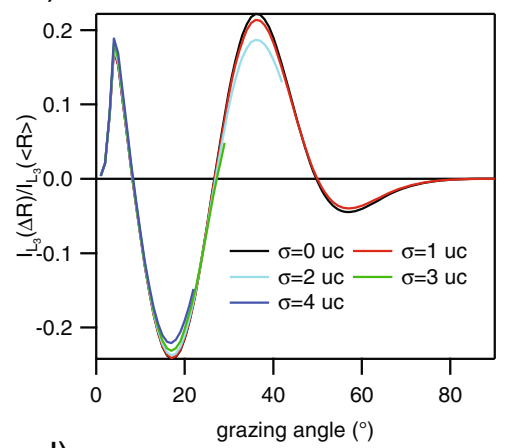

d)

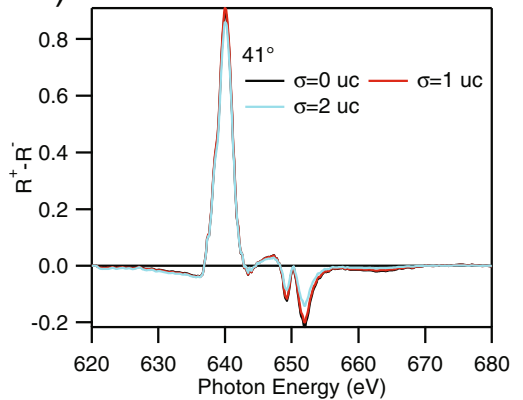

Fig. 4. Simulations of the XRMR for the case of a dead layer 4 uc thick, with both structural and magnetic roughness and different values of rms roughness $\sigma$ ranging from 0 to 4 uc. (a) Integral of the $\mathrm{L}_{3}$ peak of the averaged reflectivity $\bar{R}$ as a function of the grazing angle. (b) Ratio between the integral of the dichroic reflectivity $\Delta R$ and the integral of the averaged reflectivity $\bar{R}$ as a function of grazing angle. (c) and (d) $\Delta R$ spectra for a grazing angle of $26^{\circ}$ and $41^{\circ}$ respectively, normalized so that the difference between the maximum and the minimum value is 1 .

$I_{L_{3}}(\Delta R)$ as a function of grazing angle are reported in Fig. 4, together with the normalized XRMR spectra for $\theta=26^{\circ}$ and $41^{\circ}$.

As expected, the reflectivity strongly decreases with increasing roughness, in particular for higher grazing angle, where the diffuse scattering dominates. In practice, experimental signal-to-noise ratios will limit the normalized reflectivity to $\sim 10^{-11}$ of the value at the critical angle for a typical undulator beamline with high photon flux in this energy regime. This sets the effective lower limit for calculations in Fig. 4 (a). For example, for a roughness of $\sigma=1 \mathrm{uc}$, the reflectivity can be measured for angles up to normal incidence, while for a roughness of $\sigma=4 \mathrm{uc}$ it can only be reasonably measured for incidence angles $<25^{\circ}$. The normalized integral of $\Delta R$, shown in Fig. 4(b), has the same shape for different values of roughness (in particular, the zeroes remain unchanged) over the range of incidence angles for which comparison is possible. From Figs. 4(c) and (d), it is clear that the shape of the various dichroic spectra for different roughness can almost be superimposed at incidence angles of $26^{\circ}$ and $41^{\circ}$ after normalization.

Thus, the presence of morphological roughness (with equal rms magnetic roughness) severely reduces the range of incidence angles that can be experimentally measured, limiting the application of XRMR experiments to samples with rms roughness of less than 2-3 unit cells in our case. Structural roughness is immediately recognizable by a decrease in average reflected intensity with increasing incidence angle 
a)

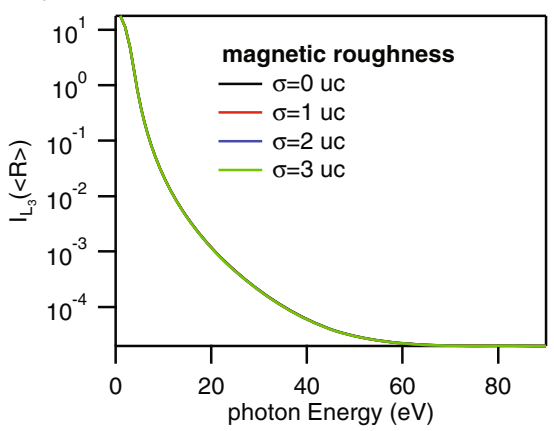

C)

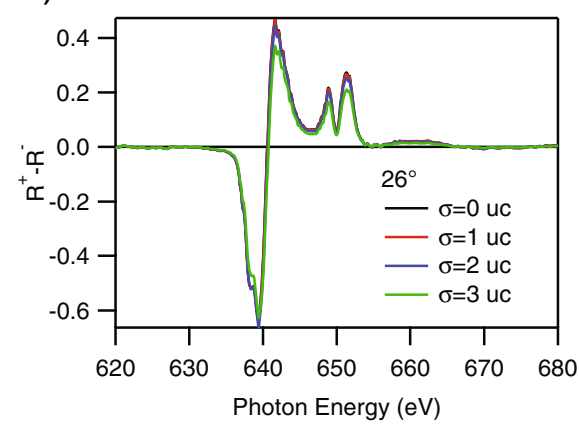

b)

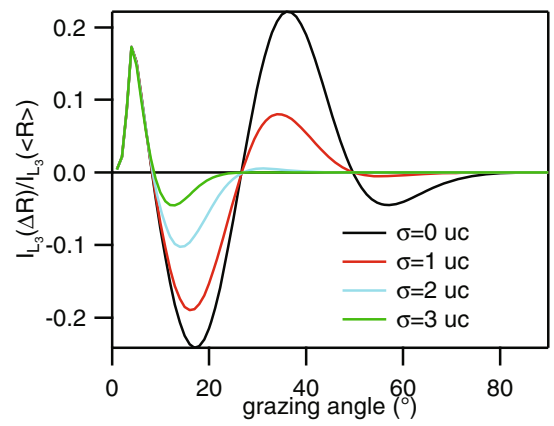

d)

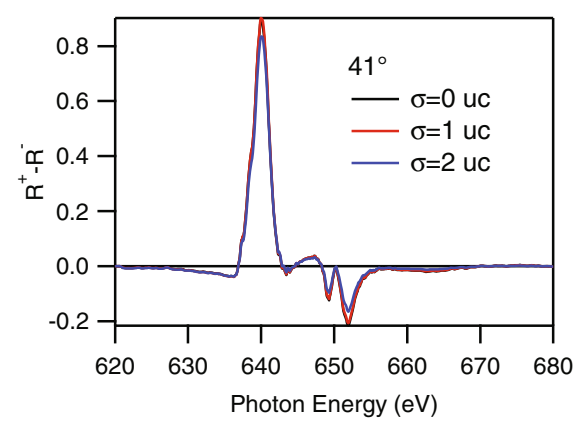

Fig. 5. Simulations of the XRMR for the case of a dead layer 4 uc thick, with only magnetic roughness (cf. Fig. 1(b)) for different values of rms roughness $\sigma$ ranging from 0 to 3 unit cells. (a) Integral of the $\mathrm{L}_{3}$ peak of the averaged reflectivity $\bar{R}$ as a function of the incidence angle. (b) Ratio between the integral of $\Delta R$ and the integral of the averaged reflectivity as a function of grazing angle. (c) and (d) $\Delta R$ spectra for a grazing angle of $26^{\circ}$ and $41^{\circ}$, respectively, normalized so that the difference between the maximum and the minimum value is 1 .

much more rapidly than the well-known $\sin ^{-4}(\theta)$ dependence for an atomically-flat surface (see Fig. 4(a)). In a simple system like this, composed just by a chemical and a magnetic interface, morphological roughness reduces the amplitude of both the average and dichroic reflectivities, leaving the shape of the dichroic signal essentially unchanged.

The case shown in Fig. 1(b) assumes an atomically flat surface, with rms magnetic roughness $\sigma$ between 0 and 3 uc. Simulations were performed of the average and normalized dichroic reflectivities, and the integrals $I_{L_{3}}(\bar{R})$ and $I_{L_{3}}(\Delta R)$ as a function of grazing angle are reported in Fig. 5, together with the normalized XRMR spectra for $\theta=26^{\circ}$ and $41^{\circ}$.

As expected, because we have not taken into account higher-order magnetooptical effects, the presence of magnetic roughness does not affect the averaged (i.e., nonmagnetic) reflectivity (cf. Fig. 5(a)) but it does significantly affect the magnitude of the dichroic reflectivity. The decrease of the dichroic signal is much more pronounced than in the case of structural roughness, and immediately allows one to distinguish between the two types of roughness. In a first-order approximation, magnetic roughness does not diffuse the scattered radiation out of the specular direction, but it strongly decreases the difference in reflectivity for the two different polarization states as the grazing angle is increased. In addition, as was previously seen in 
a)
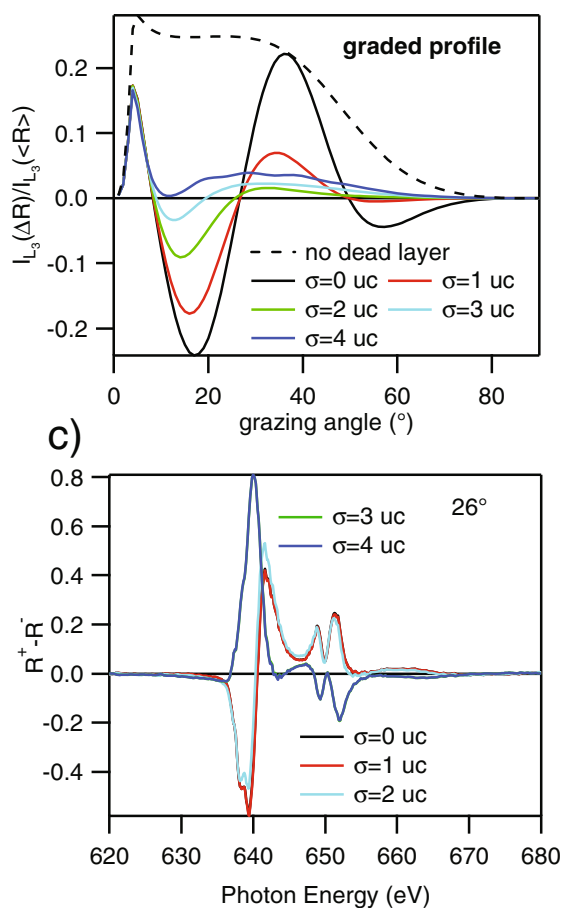

b)
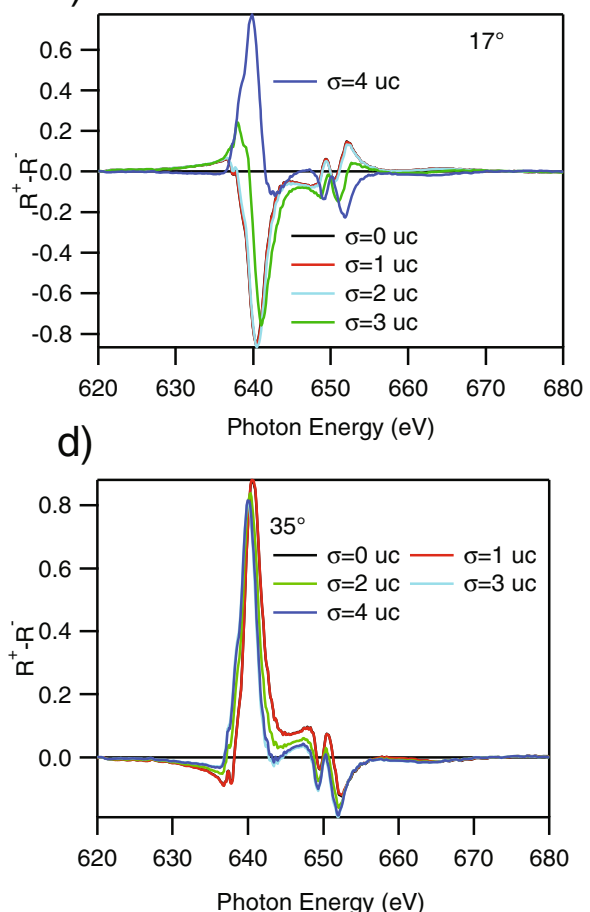

Fig. 6. Simulations of the XRMR for the case of a dead layer 4 uc thick, with a graded magnetic profile (cf. Fig. 1(c)) having different values for the smoothing parameter $\sigma$ ranging from 0 to 4 uc. (a) Ratio between the integral dichroic reflectivity $\Delta R$ and the integral of the averaged reflectivity $\bar{R}$ as a function of incidence angle. The ratio for a sample with no dead layer is also reported for comparison. (b), (c), and (d) $\Delta R$ spectra for a grazing angle of $17^{\circ}, 26^{\circ}$ and $35^{\circ}$, respectively, normalized so that the difference between the maximum and the minimum value is 1 .

Fig. 4, increasing the magnetic roughness does not change the shape of the dichroic reflectivity, as shown for two different incidence angles in Fig. 5(c) and (d).

Thus, the presence of magnetic roughness does not affect the average reflectivity of the sample, but it strongly decreases the magnitude of the normalized dichroic reflectivity as the incidence angle increases. As in the previous case (Fig. 1(a)), the shape of the dichroic reflectivity at different incidence angles is almost unaffected by roughness in the magnetic interface.

The case shown in Fig. 1(c) assumes a graded magnetization profile with smoothing parameter $\sigma$. Simulations of the average and dichroic reflectivity were performed varying $\sigma$ between $0-4$ uc (the corresponding profiles are shown in Fig. 2), and the results are shown in Fig. 6.

As before, because we did not consider higher-order contributions to magnetooptical effects, the flat sample surface results in an average reflectivity that is independent of any underlying variations in magnetization profile (not shown, but identical to Fig. 5(a) for all values of the $\sigma$ ). The effect of the graded magnetic profile, however, is readily noticeable in the dichroic reflectivity. The dependence of the normalized integrated $\Delta R$ on incidence angle is shown in Fig. 6(a). For the ensuing discussion, the dependence of the dichroic reflectivity for a sample with no dead layer ( $\mu=0 \mathrm{uc}$ ) is shown: in this case there are no interference effects and the integrated dichroic 
reflectivity is positive and decreases at higher grazing angles due to the increasing of the angle between the wavevector of the radiation $\mathbf{k}$ and the magnetization vector $\mathbf{M}$ [13]. As the smoothness parameter $\sigma$ increases from 0 to $4 \mathrm{uc}$, the simulations show that the oscillations in the integrated dichroic reflectivity weaken considerably, and for $\sigma \geq 4 \mathrm{uc}$ its integral is always positive. As the magnetization profile becomes increasingly smooth and the dead layer vanishes, the curve tends to be similar in shape to the curve indicated for no dead layer.

The disappearance of the oscillations in the integrated dichroic reflectivity with incidence angle is correlated to a change in the shape of the dichroic reflectivity at larger angles that depends on $\sigma$, as seen in the dichroic reflectivity spectra at different incidence angles in Fig. 6(b)-(d). The integrated $\mathrm{L}_{3}$ dichroic reflectivity for a perfectly sharp dead layer interface of 4 uc performs two oscillations in its sign, corresponding to three zeroes in its integral at incidence angles $\simeq 8^{\circ}, 26^{\circ}$ and $50^{\circ}$. For convenience, we denote these angles as "inversion angles", i.e., the angles at which the integral of $\Delta R$ inverts its sign.

At $\theta=8^{\circ}$ (not shown), corresponding to the first inversion angle for the curves with $\sigma=0-3 \mathrm{uc}$, there is no significant variation in the shape of the dichroic reflectivity with the parameter $\sigma$, even for $\sigma=4$ uc. At $\theta=17^{\circ}$ (Fig. 6(b)), corresponding to the maximum negative value for the integral dichroic reflectivity with $\sigma=0$, the $\mathrm{L}_{3}$ peak for $\sigma=0-2 \mathrm{uc}$ is completely negative. In contrast, the $\mathrm{L}_{3}$ peak for $\sigma \geq 3 \mathrm{uc}$ is never completely negative. At $\theta=26^{\circ}$ (Fig. $6(\mathrm{c})$ ), the $\mathrm{L}_{3}$ dichroic reflectivity for sharp enough interfaces $(\sigma=0-2 \mathrm{uc})$ has its second "inversion angle" where the integral is again zero; but for higher values of $\sigma$ the integral is positive. At $\theta=35^{\circ}$ (Fig. 6(d)), corresponding to the maximum positive value for the integral dichroic reflectivity for the sharp profile, the spectra for all $\sigma$ have essentially the same shape.

Thus, the degree of smoothing of magnetization profile can be determined by the presence and position of the inversion angles in the integrated dichroic reflectivity as a function of incidence angle, as well as the amplitude of these oscillations. A smoothed profile significantly suppresses the oscillations of the integrated dichroic reflectivity, which tends towards a positive value out to very high angles.

\section{Discussion and conclusions}

Comparison of the results shown in Figs. 4, 5 and 6 indicates that different sorts of roughness will influence the XRMR spectra in distinct ways, in most cases allowing them to be separately identified. We can draw two essential conclusions from the above results.

First, to distinguish between case 1(b) and (c), XRMR spectra need to be measured to high incidence angles, up to the third inversion angle or $50^{\circ}$ for a dead layer of 4 uc. Since the interference effect underlying the oscillations in integrated dichroic reflectivity is inversely proportional to the dead layer thickness, a thinner dead layer will require measurement out to even higher incidence angles. This implies that an XRMR study whose intent is to identify the magnetization profile of the dead layer, is necessarily limited to surfaces with rms roughness of at most 1 or 2 unit cells; in practice, this roughness should be less than one unit cell. Such roughness can be independently confirmed by, e.g., atomic force microscopy measurements, as mentioned earlier, and should be performed before any XRMR experiment is undertaken. This places strict constraints on sample surface quality, but are well within the capabilities of current state-of-the-art film growth methods for many technologically interesting materials' systems.

Second, for the study described here with a 4 uc dead layer, the XRMR technique can distinguish between rms magnetic roughness (Fig. 1(b)) and a graded magnetic 
profile (Fig. 1(c)) only for $\sigma \geq 2$, approximately, and the spectra much be taken out to angles at least $\theta \approx 45^{\circ}$, preferably to higher angles. This can be understood by noting the similarity between the integrated dichroic signal for $\sigma=1$ in Figs. 5(b) and 6(a) (they are more or less indistinguishable), whereas the curves for $\sigma=2$ show a small but experimentally measurable difference for the two cases for $\theta>30^{\circ}$. Indeed, the integrated dichroic reflectivity with magnetic roughness shown in Fig. 5(b) for $\sigma \geq 2$ uc vanishes completely above the second inversion angle; for the graded profile shown in Fig. 5(a), the integrated dichroic reflectivity for $\sigma \geq 2 \mathrm{uc}$ is positive above the second inversion angle. The difference between the two cases is much more pronounced for $\sigma=3 \mathrm{uc}$ and $\sigma=4 \mathrm{uc}$, and leaves little doubt at these higher values of $\sigma$. For the specific case investigated here (a 4 uc dead layer in LSMO), a graded profile with a smoothing parameter $\sigma \geq 3$ will present a finite magnetization in the last $\mathrm{MnO}_{2}$ plane at the surface.

B.A.D. and A.V. acknowledge support from the FVG Regional Project SPINOX funded by Legge Regionale 26/2005 and the decreto 2007/LAVFOR/1461. Authors are thankful to George Kourousias and Alessio Curri, Scientific Computing team of the IT group at Elettra synchrotron, for their help in the installation and use of PPM program.

\section{References}

1. M. Getzlaff, Fundamentals of Magnetism (Springer, Berlin, 2008)

2. R. Zhang, R.F. Willis, Phys. Rev. Lett. 86, 2665 (2001)

3. G.P. Felcher, J. Appl. Phys. 87, 5431 (2000)

4. D. Niebieskikwiat, L.E. Hueso, J.A. Borchers, N.D. Mathur, M.B. Salamon, Phys. Rev. Lett. 99, 247207 (2007)

5. J.W. Freeland, K.E. Gray, L. Ozyuzer, P. Berghuis, E. Badica, J. Kavich, H. Zheng, J.F. Mitchell, Nat. Mat. 4, 62 (2005)

6. S. Valencia, A. Gaupp, W. Gudat, Ll. Abad, Ll. Balcells, B. Martinez, J. Appl. Phys. 104, 023903 (2008)

7. J.M. Tonnerre, M. De Santis, S. Grenier, H.C.N. Tolentino, V. Langlais, E. Bontempi, M. García-Fernández, U. Staub, Phys. Rev. Lett. 100, 157202 (2008)

8. S.D. Brown, L. Bouchenoire, P. Thompson, R. Springell, A. Mirone, W.G. Stirling, A. Beesley, M.F. Thomas, R.C.C. Ward, M.R. Wells, S. Langridge, S.W. Zochowski, G.H. Lander, Phys. Rev. B 77, 014427 (2008)

9. J.B. Kortright, Sang-Koog Kim, Phys. Rev. B 62, 12216 (2000)

10. A. Verna, B.A. Davidson, Y. Szeto, A.Yu. Petrov, A. Mirone, A. Giglia, N. Mahne, S. Nannarone, J. Mag. Mag. Mat. 322, 1212 (2010)

11. A. Verna, B.A. Davidson, A.Yu. Petrov, A. Mirone, F. Yakhou-Harris, P. Orgiani, N. Mahne, A. Giglia, S. Nannarone (unpublished)

12. J.W. Freeland, V. Chakarian, K. Bussmann, Y.Z. Idzerda, H. Wende, C.-C. Kao, J. Appl. Phys. 83, 6290 (1998)

13. D.R. Lee, S.K. Sinha, D. Haskel, Y. Choi, J.C. Lang, S.A. Stepanov, G. Srajer, Phys. Rev. B 68, 224409 (2003)

14. M. Elzo, E. Jal, O. Bunau, S. Grenier, Y. Joly, A.Y. Ramos, H.C.N. Tolentino, J.M. Tonnerre, N. Jaouen, J. Mag. Mag. Mat. 324, 105 (2012)

15. A. Yu Petrov, X. Torrelles, A. Verna, H. Xu, A. Cossaro, M. Pedio, J. Garcia-Barriocanal, G.R. Castro, B.A. Davidson (unpublished)

16. https://forge.epn-campus.eu/projects/ppm/wiki

17. D.W. Berreman, J. Opt. Soc. Am. 62, 502 (1972)

18. P. Bertrand, C. Hermann, G. Lampel, J. Peretti, V.I. Safarov, Phys. Rev. B 64, 235421 (2001)

19. D.W. Windt, Comput. Phys. 12, 360 (1998) 
20. A. Gibaud, G Vignaud, in X-Ray and Neutron Reflectivity, edited by J. Daillant, A. Gibaud (Springer, Berlin, 2009)

21. C.T. Chen, Y.U. Idzerda, H.-J. Lin, N.V. Smith, G. Meigs, E. Chaban, G.H. Ho, E. Pellegrin, F. Sette, Phys. Rev. Lett. 75, 152 (1995)

22. F. Bassani, M. Altarelli, in Handbook of Synchrotron Radiation, edited by E.E. Koch (North-Holland, Amsterdam, 1983) 